\title{
Ciliobrevins as tools for studying dynein motor function
}

\author{
Douglas H. Roossien ${ }^{1}$, Kyle E. Miller ${ }^{2 *}$ and Gianluca Gallo ${ }^{3 *}$ \\ ${ }^{1}$ Department of Cell and Developmental Biology, University of Michigan, Ann Arbor, MI, USA, ${ }^{2}$ Department of Integrative \\ Biology, Michigan State University, East Lansing, MI, USA, ${ }^{3}$ Department of Anatomy and Cell Biology, Shriners Hospitals \\ Pediatric Research Center, Temple University School of Medicine, Philadelphia, PA, USA
}

Dyneins are a small class of molecular motors that bind to microtubules and walk toward their minus ends. They are essential for the transport and distribution of organelles, signaling complexes and cytoskeletal elements. In addition dyneins generate forces on microtubule arrays that power the beating of cilia and flagella, cell division, migration and growth cone motility. Classical approaches to the study of dynein function in axons involve the depletion of dynein, expression of mutant/truncated forms of the motor, or interference with accessory subunits. By necessity, these approaches require prolonged time periods for the expression or manipulation of cellular dynein levels. With the discovery of the ciliobrevins, a class of cell permeable small molecule inhibitors of dynein, it is now possible to acutely disrupt dynein both globally and locally. In this review, we briefly summarize recent work using ciliobrevins to inhibit dynein and discuss the insights ciliobrevins have provided about dynein function in various cell types with a focus on neurons. We temper this with a discussion of the need for studies that will elucidate the mechanism of action of ciliobrevin and as well as the need for experiments to further analyze the specificity of ciliobreviens for dynein. Although much remains to be learned about ciliobrevins, these small molecules are proving themselves to be valuable novel tools to assess the cellular functions of dynein.

Keywords: axon, growth cone, tension, microtubule, transport

\section{Introduction}

Motor proteins are a class of specialized enzymes that transform the chemical energy stored in ATP molecules to mechanical energy. There are three superfamilies of motor proteins (kinesins, myosins, and dyneins), which together total over 100 different motor proteins in humans (Vale, 2003). Though they vary in function, they are all closely associated with cytoskeletal filaments. Dyneins are ATPases which bind microtubules and walk toward the minus end (Sale and Satir, 1977; Paschal and Vallee, 1987; Paschal et al., 1987). Dyneins are divided into three classes. Axonemal dynein is found between microtubule doublets in cilia and flagella and powers microtubule sliding during motility. Cytoplasmic dynein contains two classes, one of which drives transport along microtubules in cilia and flagella (referred to as class 2 or IFT dynein) and another found throughout the remainder of the cell with a variety of functions (class 1) (Vale, 2003). Unless otherwise specified cytoplasmic dynein 1 will herein be referred to as dynein.

Research on the structure and mechanism of dynein function has lagged behind that of the kinesin and myosin motor superfamilies because of its large size and the large number of closely associated regulatory proteins. Recent advances in solving dynein crystal structures have begun 
to reveal the mechanochemical mechanism beyond what was previously inferred from functional studies. The motor head of dynein is made up of six ATPase domains arranged in a ring (Neuwald et al., 1999). Across this lies a linker domain that changes shape based on the status of ATP binding (Burgess et al., 2003; Kon et al., 2005, 2012; Imamula et al., 2007; Roberts et al., 2009). This linker domain is contiguous with a tail domain, which interacts with a wide variety of regulatory proteins and cargo (Figure 1). A coiled-coil stalk domain emanates from out of the motor head ring and contains a microtubule binding domain (MTBD) at its tip (Gee et al., 1997). When ATP binds to the motor head, the linker domain rotates like a hinge (Roberts et al., 2012) and the MTBD detaches from the microtubule (Porter and Johnson, 1983). ATP hydrolysis then occurs causing the MTBD to bind in a new position further along the microtubule toward the minus end (Carter et al., 2008), which in turn induces ADP $+\mathrm{P}_{\mathrm{i}}$ release (Holzbaur and Johnson, 1989). This last step in the cycle causes the linker domain to straighten and pull its attached cargo forward as the "power stroke" step (Kon et al., 2005; Kikkawa, 2013) and the mechanochemical cycle can begin again (Figure 2).

How dynein achieves long distance processivity is still unclear, though several lines of evidence suggest dimerization of two heavy chains is responsible for ensuring the motors do not dissociate from the microtubule (Reck-Peterson et al., 2006). The proposed mechanism for dimer function is that stepping of the two dynein heavy chains is uncoordinated and that the presence of the linker between the two increases the probability each will take forward steps as opposed to backward, such that the net result is forward movement of the dimer in $8 \mathrm{~nm}$ increments (Reck-Peterson et al., 2006; Dewitt et al., 2012; Qiu et al., 2012). This does not, however, account for the observation that individual heavy chains can processively take $8 \mathrm{~nm}$ steps under high load (Mallik et al., 2004), the mechanism of which remains controversial.

Unlike the kinesin and myosin superfamilies, each of which have evolved a large number of subfamilies and isoforms designed to perform specific functions in the cell, the dynein superfamily contains relatively few types (Vale, 2003). Instead, the cell utilizes accessory proteins to adapt the dynein motor complex to numerous cellular functions (Figure 1) (Vallee et al., 2012; Pfister, 2015). These include non-catalytic subunits of the dynein holoenzyme itself; two dynein light chains, a light intermediate chain, and an intermediate chain (DIC). These non-catalytic subunits mostly regulate binding to additional regulatory proteins and cargo (Roberts et al., 2013). Dynactin is a regulatory protein required for long distance dynein-driven transport of materials in living cells (Gill et al., 1991; Schroer and Sheetz, 1991). It is the most commonly studied dynein regulator and is required for almost all known functions of dynein in vivo (Schroer, 2004).

Dynactin itself is a large multi-protein complex. The major subunit, p150 Glued, binds to DIC to maintain an intact dyneindynactin complex (Karki and Holzbaur, 1995; Vaughan and Vallee, 1995). It can also bind microtubules at its N-terminus (Waterman-Storer et al., 1995; Vaughan et al., 2002). This interaction may keep dynein tethered to the microtubule to increase processivity (King and Schroer, 2000). In addition to direct microtubule binding, p150 Glued can interact with the microtubule +TIP proteins EB1 and CLIP-170 (Valetti et al., 1999; Vaughan et al., 1999, 2002). Though the precise mechanism is not yet understood, the interactions between dynactin and CLIP-170 target dynein specifically to the plus ends of microtubules, where it can remain in position until cargo binds for transport toward the minus end (Vaughan et al., 2002). Alternatively, the plus end complex can be targeted to the cell cortex along with dynein via Num1 (Markus et al., 2009) or IQGAP1 (Fukata et al., 2002). Lastly, dynactin directly binds the actin related proteins Arp1 and Arp11 (Karki et al., 2000; Eckley and Schroer, 2003), which target the dynein complex to the pointed ends of actin filaments (Eckley et al., 1999; Eckley and Schroer, 2003). Dynactin thus augments dynein function by both increasing the processivity of the motor and regulating its localization.

In addition to localization and cargo binding, dynein regulators can also directly affect motor function. BicD, for example, can increase the velocity of minus-end directed movement of vesicles, though the mechanism is still unknown (Schlager et al., 2014). Originally identified as genes causing brain developmental disorders such as lissencephaly when mutated, Lis1 and NudE have since been shown to have roles in central

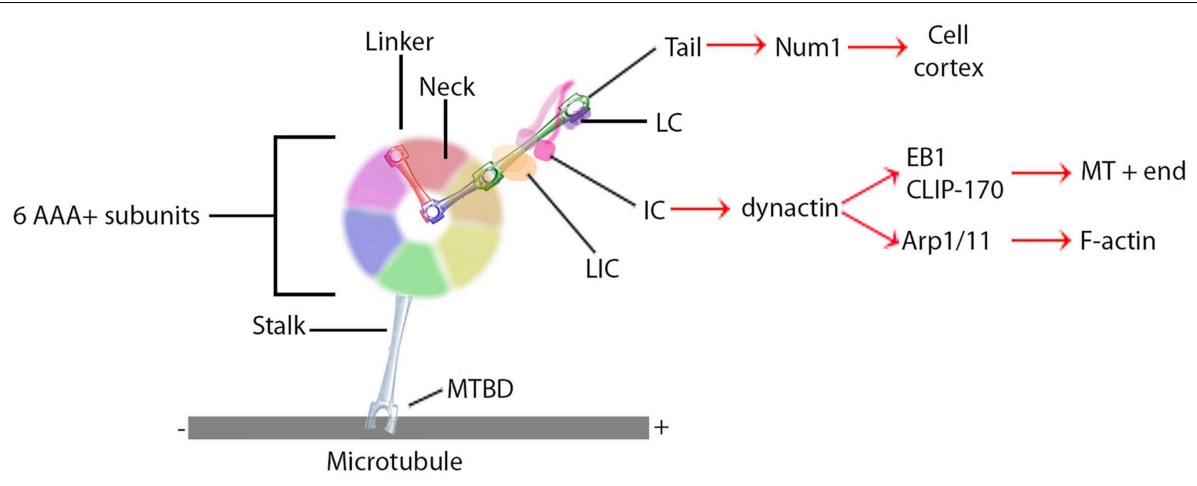

FIGURE 1 | General dynein complex structure and binding interactions. Black lines identify components of the core dynein complex whereas red arrows signify binding proteins or localizations. MTBD, microtubule binding domain; LIC, light intermediate chain; IC, intermediate chain; LC, light chain. 


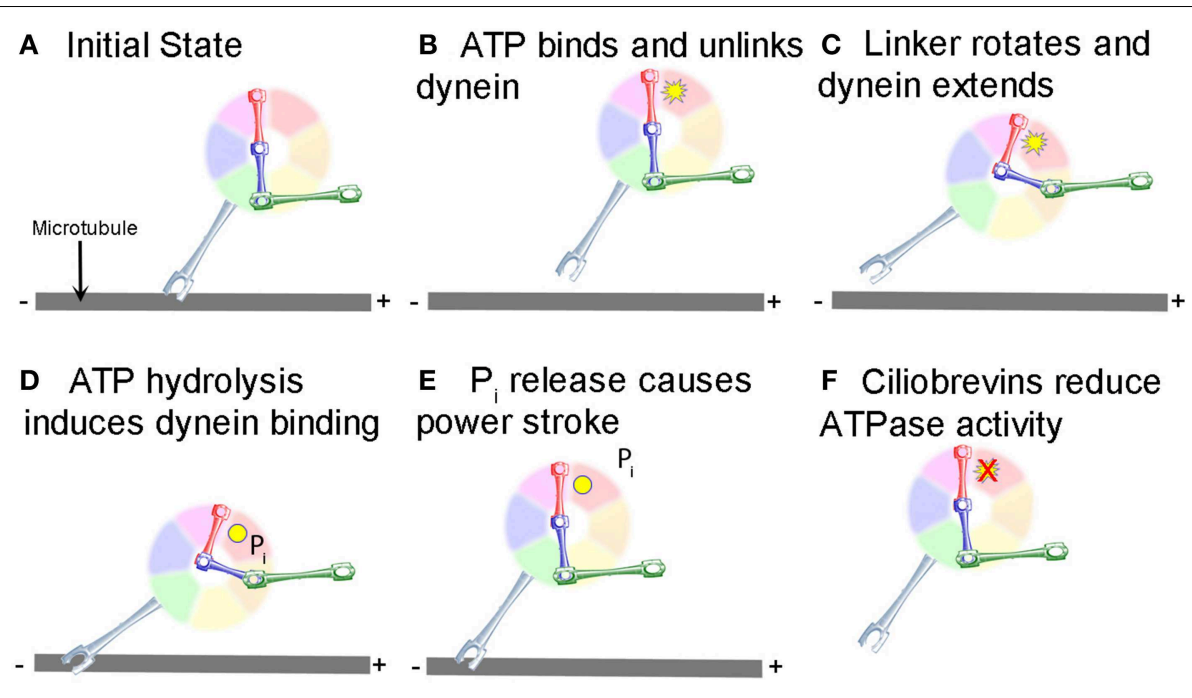

FIGURE 2 | Mechanochemical cycle of dynein. (A) Dynein is bound to the microtubule in the initial state. (B) ATP, represented by the yellow starburst, binds to the AAA+ ATPase domain, causing the MTBD to release from the MT. (C) Unlinking of the stalk from the MT causes a conformational change in the linker, which rotates and extends the stalk closer to the minus end of the MT. (D) Hydrolysis of ATP to ADP.P $P_{i}$ induces binding of the MTBD to the new location on the MT (ADP shown as a yellow circle). (E) Release of $\mathrm{P}_{\mathrm{i}}$ from the motor causes the "powerstroke" and pulls the tail and any attached cargo closer to the minus end of the MT. (F) Ciliobrevins, as indicated by the red $X$, inhibit dynein ATPase activity blocking the step shown in panels (C,D). While this is known to inhibit the motor cycle, how this occurs remains unclear. cell biological processes involving the microtubule cytoskeleton through their interactions with dynein. Lis1 is unique in its ability to bind directly to the motor domain (Figure 1), where it is recruited by NudE to produce a slow yet persistent microtubulebound force generating dynein state (Yamada et al., 2008; McKenney et al., 2010; Torisawa et al., 2011). Another recent advance in solving three-dimensional crystal structure has shown that Lis1 binds to the AAA + ring and sterically prevents the linker from completing its conformational change (Toropova et al., 2014). Lis1 has thus been described as a "clutch" (Huang et al., 2012) that keeps the motor coupled to the microtubule yet still able to generate force. In a sense, Lis1 can convert dynein from a mobile cargo transporter to a stable force-producing machine.

\section{Dynein Function in Cells}

The best characterized function of dynein is for cellular transport. In vitro, purified dynein bound to coverslips can move microtubules and dynein in solution can transport plastic beads across stationary microtubules (Lye et al., 1987; Paschal et al., 1987; Euteneuer et al., 1988). In living cells and axons dynein moves membranous vesicles toward microtubule minus ends (Schnapp and Reese, 1989; Schroer et al., 1989). Dynein mediates the transport and cellular distribution of various membranous organelles, such as mitochondria (Pilling et al., 2006), endosomes (Aniento et al., 1993; Driskell et al., 2007), and Golgi (CorthesyTheulaz et al., 1992). Because most microtubules in the axon are oriented with their minus ends directed toward the cell body, dynein drives axonal transport of membranous organelles in the retrograde direction (Schnapp and Reese, 1989; Pilling et al.,
2006; Yi et al., 2011). Dynein also transports short microtubules in the axon via Stop-and-Go transport, though in the anterograde direction (Ahmad et al., 1998, 2006). This is proposed to occur because the dynein motor domain is bound to a short microtubule while the cargo-binding domain is anchored to a structure with more resistance, such as the cross-linked bundle of microtubules or, alternatively, the cortical actin meshwork (Pfister, 1999; Baas et al., 2006). The latter presumably occurs through the interaction between dynactin and Arp1/11. Thus, dynein plays a critical role in the transport of a variety of different types of cargo in the axon and in non-neuronal cells. In all of these cases, the mechanochemical cycle brings the motor head closer to the minus end of the microtubule regardless of whether the microtubule or motor head is stationary.

Other cellular functions have recently been ascribed to dynein in addition to cellular transport, all of which rely on dynein force generation. During mitosis, dynein is required for proper mitotic spindle assembly and alignment (O'connell and Wang, 2000; Rusan et al., 2002; Goshima et al., 2005; Nguyen-Ngoc et al., 2007), capture and alignment of chromosomes (Schmidt et al., 2005), and separation of the centrosomes (Gonczy et al., 1999). Dynein at the actin-rich cortex is presumed to drive a majority of these mitotic functions. By using cortical actin as an anchor, the tendency of the motor to walk toward the minus end moves the microtubule in the plus end direction (Hendricks et al., 2012; Mazel et al., 2013). There is also a growing body of evidence in large cells, such as Zebrafish and Xenopus zygotes, that dynein is anchored in the cytoplasm to exert forces on large microtubule arrays (Kimura and Onami, 2005; Wuhr et al., 2010).

Most studies on dynein function in axons have focused on transport, be it Stop-and-Go transport of microtubules or 
retrograde transport of organelles. There have been, however, a few notable observations made of dynein function in the context of axonal elongation. Overexpression or injection of the dynactin subunit dynamitin disrupts dynein function by dissociating the dynein-dynactin complex (Echeverri et al., 1996; Wittmann and Hyman, 1999). When injected into weakly adhered neurons, the axons lose their ability to resist forces generated by non-muscle myosin II and undergo retraction (Ahmad et al., 2000). Depletion of dynein heavy chain by siRNA makes axons more sensitive to retraction induced by nitric oxide and disrupts growth cone turning (Myers et al., 2006). The rate of axon elongation is drastically reduced with both of these means of dynein disruption as well (Ahmad et al., 2000; Myers et al., 2006). In neurons grown on poly-amine substrates and then treated with soluble laminin, which increases the rate of axon elongation (Lein et al., 1992), there is a dramatic relocalization of dynein to the leading edge of the growth cone that correlates with increases in the rate of elongation (Grabham et al., 2007). These studies indicate that dynein contributes to axonal elongation, in addition to driving retrograde axonal transport. While the importance of dynein to the process of axonal elongation is well-accepted, a difficult issue has been to determine if the primary importance of dynein lies in the sustained delivery of components or if it plays additional roles in axon elongation at the growth cone or along the axon shaft. The primary obstacle to resolving this has been a lack of tools whereby dynein motor function can be acutely and locally disrupted.

\section{Ciliobrevins Inhibit the Motor Activity of Cytoplasmic Dynein}

Ciliobrevins are a group of small molecules recently determined to be inhibitors of the motor activity of dynein 1 and 2 (Firestone et al., 2012). The discovery of ciliobrevins begun with the identification of a benzoyl dihydroquinazolinone (HPI-4) in a screen of compounds with the ability to impair cellular effects downstream of smoothened signaling in the hedgehog signaling pathway (Hyman et al., 2009). Interestingly, prolonged treatment with HPI-4 was also observed to decrease the number and length of cilia. To follow up on this observation, Firestone et al. (2012) synthesized analogs of HPI-4 and found a group that inhibited hedgehog signaling but did not affect cilia, and one group which affected both. The latter group was termed the ciliobrevins (A-D). The hedgehog pathway drives the accumulation of a signaling component termed Gli2 at the tip of cilia. Ciliobrevin A and D were found to mimic the effects of the N-terminal domain of hedgehog on the targeting of Gli2 to the tips of cilia. This observation suggested to Firestone et al. (2012) that ciliobrevins may be affecting aspects of the intra-flagellar/cilial transport of Gli2. Kinesins and dynein are respectively considered to mediate the anterograde and retrograde transport of flagellar/cilia components. Loss of dynein 2 had been previously reported to result in increased levels of Gli2 in cilia, as observed with ciliobrevins, suggesting ciliobrevins may be interfering with dynein function. Using in vitro reconstituted systems for tracking the motor activity of dynein, as reported by the motor protein's ability to move assembled microtubules, ciliobrevin $\mathrm{A}$ and $\mathrm{D}$ were found to block dynein activity. In contrast, ciliobrevins had no effect on kinesin1 mediated microtubule movements. Analysis of the ATPase activity of dynein and kinesin 1 and 5 revealed that ciliobrevin $\mathrm{D}$ affects dynein ATPase function, but not the tested kinesins. Hanes-Woolf analysis indicates that ciliobrevins may act as ATP competitors for the dynein ATPase (Figure 2F). Ciliobrevins did not affect the binding of dynein to microtubules in the ADPloaded state. Ciliobrevins were also shown to be effective in living cells, and blocked a variety of phenomena considered to be dependent on dynein 1 or 2. Ciliobrevins inhibited melanosome aggregation in Xenopus melanophores, peroxisome movement in insect cells, and disrupted spindle pole assembly and kinetochore microtubule attachment in NIH-3T3 and HeLa cells. Collectively, the work by Firestone et al. (2012) provides compelling evidence that ciliobrevins $\mathrm{A}$ and $\mathrm{D}$ are effective inhibitors of dynein activity and can be used to assay dynein functions in living cells.

\section{Utilization of Ciliobrevins to Probe the Functions of Dynein in Non-neuronal Cells}

Since their initial discovery and characterization, ciliobrevins have been used by multiple groups to probe the functions of dynein in living non-neuronal cells. Kim et al. (2014) used ciliobrevin A to determine the role of dynein in the targeting of smoothened into cilia. Consistent with the work of Firestone et al. (2012), ciliobrevin initially decreased the efflux of smoothened from cilia, while longer term treatments also impaired to targeting of smoothened into cilia. Cao et al. (2015) used ciliobrevin $\mathrm{D}$ to investigate the role of dynein in the targeting of the membrane protein SAG1-C65 to the periciliar domain of Chlamydomona. Sikirzhytski et al. (2014) used ciliobrevin D at sub-maximal doses to decrease, but not completely inhibit, dynein activity during mitosis. This resulted in normal bipolar spindles and chromosome congression into the metaphase plate, but increased the width of the spindle pole. Fu et al. (2014) used ciliobrevin D to inhibit cilia formation and concluded that cilia are necessary for the differentiation of muscle cells. Eyre et al. (2014) used ciliobrevin D to investigate the role of dynein in the control of the subcellular distribution of the hepatitis $\mathrm{C}$ viral protein NS5A, which is required for efficient viral RNA replication and infectious viron assembly. This study revealed that dynein is required for the intracellular traffic of NS5A and efficient viral RNA replication. Yi et al. (2013) used ciliobrevin $\mathrm{D}$ to determine that the reorientation of the centrosome toward the immunological synapse in $\mathrm{T}$ cells depends on a microtubule dependent and dynein driven mechanism. In contrast, Liu et al. (2013) found that while inhibition of dynein or myosin II alone, using both pharmacological (ciliobrevin $\mathrm{D}$ and blebbistatin, respectively) and molecular approaches, generated only mild effects on centrosome positioning toward the immunological synapse, inhibition of both motors caused much more pronounced effects.

Recent evidence indicates that dynein also has roles in the orchestration of active signaling mechanisms in cells. Clippinger 
and Alwine (2012) used ciliobrevin to determine that dynein transports mTOR to the perinuclear domain. The lack of mTOR transport results in impairment of mTOR activity due to the failure to localize it in the proximity of the upstream activator RheB. Using lymphocytes, Wang et al. (2015) investigated the role of dynein in the internalization and centripetal transport of CD40 following binding to its ligand CD154. Ciliobrevin D treatment impaired the internalization and transport of CD40, as well as the activation of the downstream MAPK pathway by CD154-CD40.

Investigations with ciliobrevins are contributing to the growing literature indicating an interdependence between anterograde and retrograde transport mechanisms. Blasius et al. (2013) addressed the role of retrograde transport mechanisms in the regulation of the accumulation of kinesin-1 motors in the distal processes of differentiated CAD cells. Acute treatment with ciliobrevin A or D decreased the levels of kinesin-1 at the distal tips of processes. This observation is counter to the expectation of increased levels of distal kinesin-1 if retrograde transport mediated the evacuation of kinesin-1 from the tips of processes, indicating that retrograde transport does not have a major role in maintaining the distal accumulation of kinesin-1. However, the observation indicates a possible impairment of the anterograde transport and delivery of kinesin1 , which was further suggested by additional considerations in Blasius et al. (2013). Similarly, Ye et al. (2013) found that ciliobrevin treatment rapidly $(2-3 \mathrm{~min})$ inhibited the intraflagellar retrograde transport of the intraflagellar transport component IFT88, but at longer times following treatment (30 min) also inhibited its anterograde transport. In a study of the role of intraflagellar transport mechanisms in cilia-mediated Chlamydomonas gliding motility, Shih et al. (2013) also observed that ciliobrevin $\mathrm{D}$ treatment affected both retrograde and anterograde intra-flagellar transport. The bidirectional effects of inhibiting dynein and treatment with ciliobrevin $\mathrm{D}$ on microtubule based transport mechanisms are further considered in the next section. Collectively, these studies emphasize the usefulness of ciliobrevins as tools for addressing the cellular functions of dynein in a variety of cellular model systems.

\section{Use of Ciliobrevins to Address the Role of Dynein in Primary Neurons}

The dynein-dependent retrograde transport of organelles and proteins from the distal axon to the cell body is a fundamental aspect of neurobiology, and the malfunction of transport mechanisms is considered to underlie aspects of neurodegenerative conditions (Ikenaka et al., 2012; Kanaan et al., 2013). Two studies on the intra-axonal translation of proteins with functions in the cell body have used ciliobrevins to block their subsequent retrograde transport. Melemedjian et al. (2014) provided evidence that IL-6 treatment of the distal axons of sensory peripheral nerves in vivo induces the intra-axonal synthesis of the transcription factor CREB, which subsequently undergoes retrograde axonal transport along the nerves and contributes to the development of pain sensitivity. In this study, ciliobrevin D was locally delivered along peripheral nerves, proximal to the site of IL- 6 treatment, and found to block the IL-6 induced pain sensitivity. Similarly, local delivery of microtubule depolymerizing drugs, which also perturb transport, blocked the development of pain sensitivity. Control experiments indicated that the effects of ciliobrevin D and microtubule depolymerizing drugs were indeed local. Similarly, Baleriola et al. (2014) report that Amyloid $\beta$-peptide 1-42, considered to contribute to the development of Alzheimer's disease, induced the intra-axonal synthesis of the transcription factor ATF4 which in turn is a component of the ensuing cell death mechanism within the neuronal cell body. Treatment with ciliobrevin A specifically to axons using microfluidic chambers prevented the retrograde transport of axonally synthesized ATF4 and decreased Amyloid $\beta$-peptide 1-42 induced cell death. These studies provide evidence that ciliobrevins provide a new venue for investigating the role of retrograde axonal transport in experimental paradigms involving acute and localized treatments.

Two studies using cultured embryonic sensory neurons report that treatment with ciliobrevin D causes the rapid, and reversible, cessation of axon extension (Roossien et al., 2014; Sainath and Gallo, 2014). Local application of ciliobrevin D to the growth cone, using microperfusion, was sufficient to stall the extension of the axon, indicating a role for dynein at the growth cone during axon extension (Roossien et al., 2014). The main axon also generates collateral branches which assist in the formation of complex circuitry. Ciliobrevin D inhibited the formation of axon branches in response to the branch inducing signal nerve growth factor (NGF; Sainath and Gallo, 2014). Roossien et al. (2014) also noted that, similar to inhibition of dynein activity through the intracellular injection of function blocking antibodies, ciliobrevin $\mathrm{D}$ treatment inhibited the en bloc translocation of the axonal microtubule array, as deduced from the motion of docked mitochondria. In the same study, treatment with ciliobrevin D also increased axon tension, providing insights into the effects of dynein inhibition on axon extension. The effects of the local and acute inhibition of dynein function, achieved through treatment with ciliobrevin in these studies, further agues for a role of dynein in regulating axon extension through mechanisms operative within the axon itself.

Interestingly, ciliobrevin $\mathrm{D}$ treatment impaired both the retrograde and anterograde transport of mitochondria (Roossien et al., 2014; Sainath and Gallo, 2014) and lysosomes and Golgiderived vesicles along axons (Sainath and Gallo, 2014). The bidirectional effects of altering dynein function on transport are not novel, and interested readers are directed to the discussion in both publications. The novelty of the observations using ciliobrevin $\mathrm{D}$ in the context of the role of dynein in bidirectional transport is that they indicate that inhibition of the ATPase activity of dynein alone may translate into bidirectional effects on transport. In contrast, a study of the initial emergence of neurites from the cell bodies of cultured Drosophila neurons failed to note effects of ciliobrevin D treatment on neurite formation ( $\mathrm{Lu}$ et al., 2013). The initial formation of neurites from the cell bodies of these neurons was found to depend on kinesin-1 driven microtubule gliding into the nascent processes. In this study ciliobrevin $\mathrm{D}$ impaired the kinesin-1 dependent 
motility of mitochondria as noted in the studies by Roossien et al. (2014) and Sainath and Gallo (2014), but did not affect kinesin-1 mediated microtubule sliding. The observation that ciliobrevin $\mathrm{D}$ affects one form of kinesin-1 driven motility, but not another, is interesting and indicates that the bidirectional effects of inhibiting dynein may not translate to all cargoes. One possibility that may explain these differences is that in some cases kinesin and dynein activity are regulated cooperatively; such that when one motor is active the other is inhibited (Miller et al., 2005; Fu and Holzbaur, 2014; Hancock, 2014). Taking this idea to its logical conclusion raises the possibility that when ciliobrevin binds to dynein it causes it to assume a physical confirmation that mimics an "active" motor and thus suppresses kinesin. Yet in other cases, there is a "tugof-war" between kinesin and dynein where the activity of the motors is not coordinated and thus disruption of dynein allows kinesin to win. Another possibility is that when kinesin and dynein work cooperatively, there is a checkpoint mechanism in place that ensures that functional kinesin and dynein are both bound to the cargo. Thus, if either kinesin or dynein are absent or dysfunctional, the checkpoint is flagged and the cargo is motionless. If this occurred ciliobrevin may inhibit bi-directional transport by either causing the disassociation of dynein from the motor complex or by causing dynein to assume a confirmation that is "non-functional." Finally, it is possible that ciliobrevin locks dynein in a confirmation where it is stably associated with microtubules but is unable to generate forces. If this occurred, the drag associated with dynein microtubule binding could decrease kinesin-based transport. These highly speculative possibilities highlight the importance of understanding the molecular mechanism of action of ciliobrevin.

The studies by Roossien et al. (2014) and Sainath and Gallo (2014) both noted that treatment with ciliobrevin D alone induces some degree of retraction of the distal axon in established axons that were actively extending. Ciliobrevin D was also found to decrease the microtubule content of distal axons (Sainath and Gallo, 2014) and mitochondria exhibited net retrograde displacements in the distal-most segment of the axon (Roossien et al., 2014). When myosin II activity was inhibited using blebbistatin (Roossien et al., 2014), or actin filaments depolymerized using latrunculin-A (Sainath and Gallo, 2014), growth cones advanced faster and underwent collapse and exhibited retrograde cytoplasmic evacuation, respectively. Interestingly, treatment with ciliobrevin $\mathrm{D}$ partially countered both of these effects. These observations are generally consistent with the notion of antagonistic relationships between the actomyosin system and dynein (Ahmad et al., 2000; Myers et al., 2006). Further suggestions that dynein may be involved in the regulation of the axonal actin cytoskeleton arise from the investigation of the reorganization of actin filaments in axons undergoing Wallerian degeneration following severing from the cell body (Sainath and Gallo, 2014). During degeneration the actin filament content of axons was found to increase, and treatment with ciliobrevin $\mathrm{D}$ prevented the increase in axonal actin filaments while promoting the morphological degeneration of axons. These observations suggest an antagonistic role for dynein in axon degeneration, possibly through the regulation of the actin cytoskeleton which may have a protective modulatory role in the timing of the ensuing degeneration.

Firestone et al. (2012) found that ciliobrevin treatment resulted in the loss of pre-existing microtubules in mitotic spindles, but did not affect microtubules in non-mitotic NIH3T3 cells. As noted above, Sainath and Gallo (2014) observed that ciliobrevin D treatment resulted in decreased levels of microtubules in the distal axon, and also altered the relative levels of tubulin post-translational modifications. While at first glance it may be surprising that the microtubules of a post-mitotic neuron would behave similarly to those of a mitotic cell in response to ciliobrevin treatment, this observation is consistent with the notion that neurons utilize aspects of the mitotic microtubule apparatus to extend their axons (Ferhat et al., 1998a,b; Buster et al., 2003; Nadar et al., 2008; Falnikar et al., 2011; Lin et al., 2012). However, Firestone et al. (2012) did not observe abnormal subcellular targeting of the p150 Glued dynactin subunit following treatment with ciliobrevins. In contrast, ciliobrevin D treatment resulted in the redistribution of $\mathrm{p} 150^{\text {Glued }}$ to linear structures in axons (Sainath and Gallo, 2014), presumably mitochondria, indicating differences in the effects of ciliobrevin treatment on the distribution of $\mathrm{p} 150^{\text {Glued }}$ in sensory neurons relative to NIH3T3 cells.

\section{Concluding Remarks}

The multifaceted functionality of dynein in neurons (Figure 3) makes it difficult to interpret the results of experiments involving the manipulation of dynein levels and function. Further complicating this problem has been the lack of reagents that are capable of acutely and locally disrupting dynein. For example, knocking down or overexpressing dynein affects the extension of axons, but because these approaches are chronic and global it has been difficult to resolve if dynein is playing a direct role in elongation at the growth cone, along the axon shaft or as the result of disrupting the trafficking of materials to and from the cell body. The strength of ciliobrevins is that they allow for acute and localized inhibition of dynein function. Nonetheless, while ciliobrevins are an attractive tool, cautionary notes ought to be taken into consideration. Dynein belongs to the AAA + ATPase family of proteins, which mediate a variety of cellular processes. Firestone et al. (2012) did not detect effects on ciliobrevins on two other AAA+ ATPase proteins (p97 and Mcm2-7). However, given the diversity of this molecular family, further scrutiny will be required to rule out potential off target effects. Similarly, continued screening for possible effects of ciliobrevins on additional motor proteins is warranted. The bidirectional effects of ciliobrevins on the axonal transport of organelles may be due to the previously noted inter-dependence of antero and retrograde mechanisms, but may also reflect off target effects. The importance of this particular question in the field underscores the need for a tool that more confidently separates the action of individual motor subtypes in an acute and local fashion. Whether the ciliobrevins accomplish this degree of specificity is still unclear and will remain a limitation of their use until further insight into their action is gained. The bidirectional effects of inhibiting dynein 


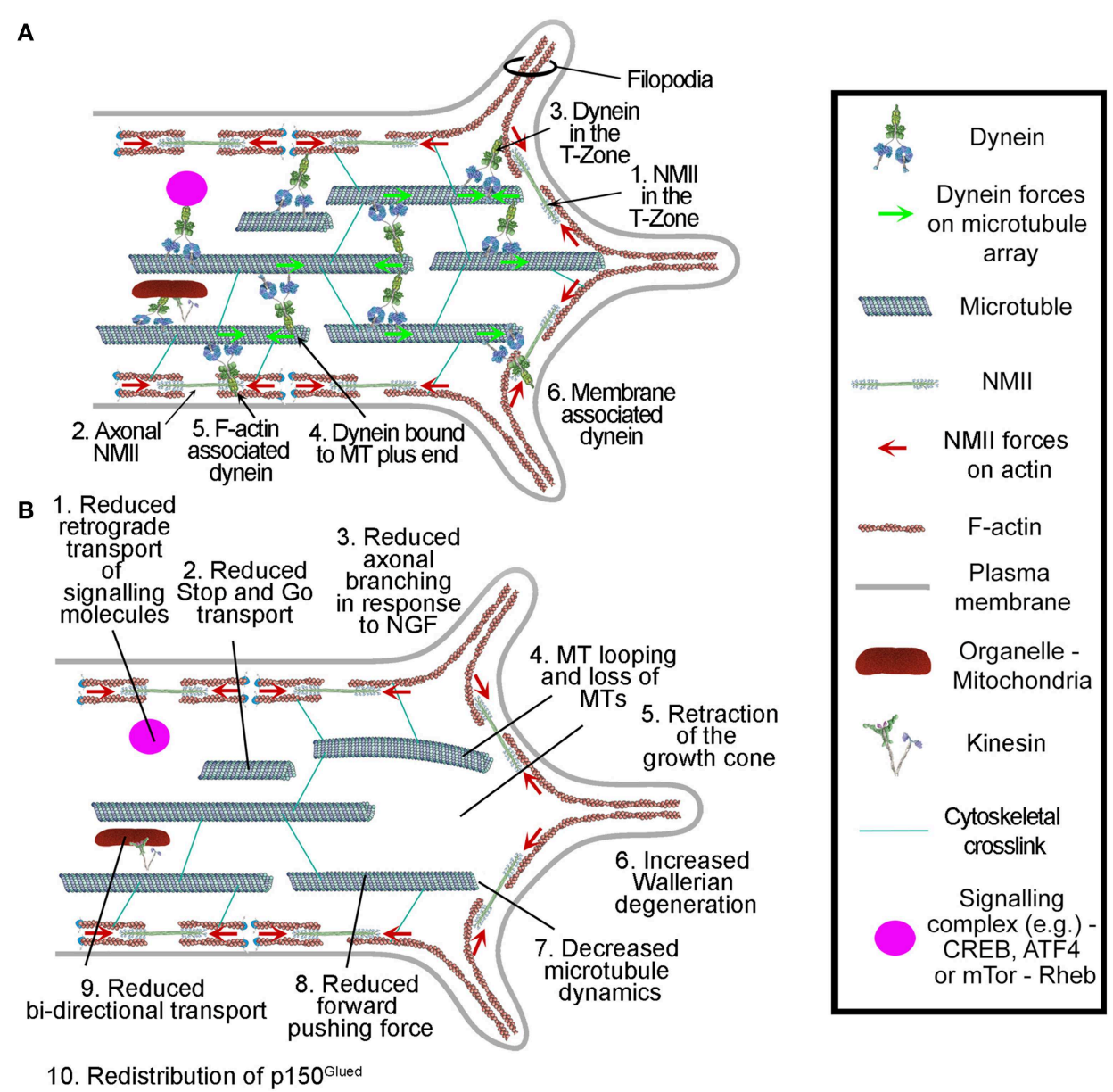

FIGURE 3 | Summary of the roles of dynein in axons and growth cones. (A) Dynein localization and interactions in axons and growth cones. (B) Reported effects of dynein depletion/inhibition on axons and growth cones. All but items 2 and 7 were observed through chronic manipulation of dynein and also using ciliobrevins.
The effects of ciliobrevins on stop-and-go transport and microtubule plus tip dynamics, items 2 and 7 , remain to be directly determined. The role for dynein in axon degeneration, item 6 , is based solely on the use of ciliobrevin $D$ and requires confirmation though additional approaches. function on transport mechanisms thus ought to be considered in the interpretation of experimental results which may be an indirect consequence of altered anterograde transport and not directly attributable to dynein per se. Alternatively, dynein can also serve to organize aspects of signal transduction mechanisms (Clippinger and Alwine, 2012; Kim et al., 2014; Wang et al., 2015). Thus, the effects of inhibiting dynein, even if acutely, may ultimately be due to its contribution to signaling mechanisms independent of its direct roles in the regulation of microtubules, an interpretational aspect that ought to be considered. For example, the inhibition of axon branching in response to NGF by ciliobrevin D (Sainath and Gallo, 2014) may in part be due to a role for dynein in organizing mTOR-Rheb signaling (Clippinger and Alwine, 2012), which is required for NGF-induced branching (Spillane et al., 2012). Continued analysis of the cellular effects of ciliobrevins will provide further insights into the degree of specificity of action of these compounds, and provide a richer landscape for the interpretation of results addressing the cellular functions of dynein. Specific important questions to answer going forward are as follows: Does ciliobrevin block the ability of ATP to bind dynein and thus leaves dynein in a nucleotide free conformation where it is bound tightly to the microtubule? Does it bind to dynein and locks it into an inactive conformation that mimics the ADP bound state, which again would cause it to associate tightly with microtubules? Does it bind to dynein and induces it to assume a transition state that mimics the active conformation, where dynein is weakly attached to the microtubule? Or does it does it bind to dynein and cause it to disassociate from the cargo? While some of these possibilities seem more plausible than others, clear answers to these questions will help in the interpretation of studies that use ciliobrevin, though it is likely that caution will continue to be needed in the interpretation of future studies. In sum, ciliobrevins are an important new tool as they now allow tight spatial and temporal manipulation of dynein. Nonetheless, ciliobrevins come with their own interpretational issues; they still remain to be fully characterized and off target effects remain a concern. 


\section{Author Contributions}

DR, KM, and GG all continued equally to the writing of this manuscript. KM and GG share senior authorship.

\section{References}

Ahmad, F. J., Echeverri, C. J., Vallee, R. B., and Baas, P. W. (1998). Cytoplasmic dynein and dynactin are required for the transport of microtubules into the axon. J. Cell Biol. 140, 391-401. doi: 10.1083/jcb.140.2.391

Ahmad, F. J., He, Y., Myers, K. A., Hasaka, T. P., Francis, F., Black, M. M., et al. (2006). Effects of dynactin disruption and dynein depletion on axonal microtubules. Traffic 7, 524-537. doi: 10.1111/j.1600-0854.2006.00403.x

Ahmad, F. J., Hughey, J., Wittmann, T., Hyman, A., Greaser, M., and Baas, P. W. (2000). Motor proteins regulate force interactions between microtubules and microfilaments in the axon. Nat. Cell Biol. 2, 276-280. doi: 10.1038/350 10544

Aniento, F., Emans, N., Griffiths, G., and Gruenberg, J. (1993). Cytoplasmic dynein-dependent vesicular transport from early to late endosomes. J. Cell Biol. 123, 1373-1387. doi: 10.1083/jcb.123.6.1373.

Baas, P. W., Vidya Nadar, C., and Myers, K. A. (2006). Axonal transport of microtubules: the long and short of it. Traffic 7, 490-498. doi: 10.1111/j.16000854.2006.00392.x

Baleriola, J., Walker, C. A., Jean, Y. Y., Crary, J. F., Troy, C. M., Nagy, P. L., et al. (2014). Axonally synthesized ATF4 transmits a neurodegenerative signal across brain regions. Cell 158, 1159-1172. doi: 10.1016/j.cell.2014.07.001

Blasius, T. L., Reed, N., Slepchenko, B. M., and Verhey, K. J. (2013). Recycling of kinesin-1 motors by diffusion after transport. PLOS ONE 8:e76081. doi: 10.1371/journal.pone.0076081

Burgess, S. A., Walker, M. L., Sakakibara, H., Knight, P. J., and Oiwa, K. (2003). Dynein structure and power stroke. Nature 421, 715-718. doi: 10.1038 /nature 01377

Buster, D. W., Baird, D. H., Yu, W., Solowska, J. M., Chauviere, M., Mazurek, A., et al. (2003). Expression of the mitotic kinesin Kif15 in postmitotic neurons: implications for neuronal migration and development. J. Neurocytol. 32, 79-96. doi: 10.1023/A:1027332432740

Cao, M., Ning, J., Hernandez-Lara, C. I., Belzile, O., Wang, Q., Dutcher, S. K., et al. (2015). Uni-directional ciliary membrane protein trafficking by a cytoplasmic retrograde IFT motor and ciliary ectosome shedding. Elife 4:e05242. doi: 10.7554/eLife.05242

Carter, A. P., Garbarino, J. E., Wilson-Kubalek, E. M., Shipley, W. E., Cho, C., Milligan, R. A., et al. (2008). Structure and functional role of dynein's microtubule-binding domain. Science 322, 1691-1695. doi: $10.1126 /$ science. 1164424

Clippinger, A. J., and Alwine, J. C. (2012). Dynein mediates the localization and activation of mTOR in normal and human cytomegalovirus-infected cells. Genes Dev. 26, 2015-2026. doi: 10.1101/gad.196147.112

Corthesy-Theulaz, I., Pauloin, A., and Pfeffer, S. R. (1992). Cytoplasmic dynein participates in the centrosomal localization of the Golgi complex. J. Cell Biol. 118, 1333-1345. doi: 10.1083/jcb.118.6.1333

Dewitt, M. A., Chang, A. Y., Combs, P. A., and Yildiz, A. (2012). Cytoplasmic dynein moves through uncoordinated stepping of the AAA+ ring domains. Science 335, 221-225. doi: 10.1126/science.1215804

Driskell, O. J., Mironov, A., Allan, V. J., and Woodman, P. G. (2007). Dynein is required for receptor sorting and the morphogenesis of early endosomes. Nat. Cell Biol. 9, 113-120. doi: 10.1038/ncb1525

Echeverri, C. J., Paschal, B. M., Vaughan, K. T., and Vallee, R. B. (1996). Molecular characterization of the $50-\mathrm{kD}$ subunit of dynactin reveals function for the complex in chromosome alignment and spindle organization during mitosis. J. Cell Biol. 132, 617-633. doi: 10.1083/jcb.132.4.617

Eckley, D. M., Gill, S. R., Melkonian, K. A., Bingham, J. B., Goodson, H. V., Heuser, J. E., et al. (1999). Analysis of dynactin subcomplexes reveals a novel actinrelated protein associated with the arp1 minifilament pointed end. J. Cell Biol. 147, 307-320. doi: 10.1083/jcb.147.2.307

\section{Acknowledgments}

This work was supported by awards NIH NS078030 (GG) and NSF IOS_0951019 (KM). NIH 1R01MH094607-01A1 (KM).

Eckley, D. M., and Schroer, T. A. (2003). Interactions between the evolutionarily conserved, actin-related protein, Arp11, actin, and Arp1. Mol. Biol. Cell 14, 2645-2654. doi: 10.1091/mbc.E03-01-0049

Euteneuer, U., Koonce, M. P., Pfister, K. K., and Schliwa, M. (1988). An ATPase with properties expected for the organelle motor of the giant amoeba, Reticulomyxa. Nature 332, 176-178. doi: 10.1038/332176a0

Eyre, N. S., Fiches, G. N., Aloia, A. L., Helbig, K. J., McCartney, E. M., McErlean, C. S., et al. (2014). Dynamic imaging of the hepatitis C virus NS5A protein during a productive infection. J. Virol. 88, 3636-3652. doi: 10.1128/JVI.02490-13

Falnikar, A., Tole, S., and Baas, P. W. (2011). Kinesin-5, a mitotic microtubuleassociated motor protein, modulates neuronal migration. Mol. Biol. Cell 22, 1561-1574. doi: 10.1091/mbc.E10-11-0905

Ferhat, L., Cook, C., Chauviere, M., Harper, M., Kress, M., Lyons, G. E., et al. (1998a). Expression of the mitotic motor protein Eg5 in postmitotic neurons: implications for neuronal development. J. Neurosci. 18, 7822-7835

Ferhat, L., Kuriyama, R., Lyons, G. E., Micales, B., and Baas, P. W. (1998b). Expression of the mitotic motor protein CHO1/MKLP1 in postmitotic neurons. Eur. J. Neurosci. 10, 1383-1393. doi: 10.1046/j.1460-9568.1998.00159.x

Firestone, A. J., Weinger, J. S., Maldonado, M., Barlan, K., Langston, L. D., O'donnell, M., et al. (2012). Small-molecule inhibitors of the AAA+ ATPase motor cytoplasmic dynein. Nature 484, 125-129. doi: 10.1038/nature10936

Fu, M. M., and Holzbaur, E. L. (2014a). Integrated regulation of motor-driven organelle transport by scaffolding proteins. Trends Cell Biol. 24, 564-574. doi: 10.1016/j.tcb.2014.05.002

Fu, W., Asp, P., Canter, B., and Dynlacht, B. D. (2014b). Primary cilia control hedgehog signaling during muscle differentiation and are deregulated in rhabdomyosarcoma. Proc. Natl. Acad. Sci. U.S.A. 111, 9151-9156. doi: $10.1073 /$ pnas.1323265111

Fukata, M., Watanabe, T., Noritake, J., Nakagawa, M., Yamaga, M., Kuroda, S., et al. (2002). Rac1 and Cdc42 capture microtubules through IQGAP1 and CLIP-170. Cell 109, 873-885. doi: 10.1016/S0092-8674(02)00800-0

Gee, M. A., Heuser, J. E., and Vallee, R. B. (1997). An extended microtubulebinding structure within the dynein motor domain. Nature 390, 636-639. doi: $10.1038 / 37663$

Gill, S. R., Schroer, T. A., Szilak, I., Steuer, E. R., Sheetz, M. P., and Cleveland, D. W. (1991). Dynactin, a conserved, ubiquitously expressed component of an activator of vesicle motility mediated by cytoplasmic dynein. J. Cell Biol. 115, 1639-1650. doi: 10.1083/jcb.115.6.1639

Gonczy, P., Pichler, S., Kirkham, M., and Hyman, A. A. (1999). Cytoplasmic dynein is required for distinct aspects of MTOC positioning, including centrosome separation, in the one cell stage Caenorhabditis elegans embryo. J. Cell Biol. 147, 135-150. doi: 10.1083/jcb.147.1.135

Goshima, G., Nedelec, F., and Vale, R. D. (2005). Mechanisms for focusing mitotic spindle poles by minus end-directed motor proteins. J. Cell Biol. 171, 229-240. doi: $10.1083 /$ jcb. 200505107

Grabham, P. W., Seale, G. E., Bennecib, M., Goldberg, D. J., and Vallee, R. B. (2007). Cytoplasmic dynein and LIS1 are required for microtubule advance during growth cone remodeling and fast axonal outgrowth. J. Neurosci. 27, 5823-5834. doi: 10.1523/JNEUROSCI.1135-07.2007

Hancock, W. O. (2014). Bidirectional cargo transport: moving beyond tug of war. Nat. Rev. Mol. Cell Biol. 15, 615-628. doi: 10.1038/nrm3853

Hendricks, A. G., Lazarus, J. E., Perlson, E., Gardner, M. K., Odde, D. J., Goldman, Y. E., et al. (2012). Dynein tethers and stabilizes dynamic microtubule plus ends. Curr. Biol. 22, 632-637. doi: 10.1016/j.cub.2012.02.023

Holzbaur, E. L., and Johnson, K. A. (1989). Microtubules accelerate ADP release by dynein. Biochemistry 28, 7010-7016. doi: 10.1021/bi00443a034

Huang, J., Roberts, A. J., Leschziner, A. E., and Reck-Peterson, S. L. (2012). Lis1 acts as a "clutch" between the ATPase and microtubule-binding domains of the dynein motor. Cell 150, 975-986. doi: 10.1016/j.cell.2012.07.022 
Hyman, J. M., Firestone, A. J., Heine, V. M., Zhao, Y., Ocasio, C. A., Han, K., et al. (2009). Small-molecule inhibitors reveal multiple strategies for Hedgehog pathway blockade. Proc. Natl. Acad. Sci. U.S.A. 106, 14132-14137. doi: 10.1073/pnas.0907134106

Ikenaka, K., Katsuno, M., Kawai, K., Ishigaki, S., Tanaka, F., and Sobue, G. (2012). Disruption of axonal transport in motor neuron diseases. Int. J. Mol. Sci. 13, 1225-1238. doi: 10.3390/ijms13011225

Imamula, K., Kon, T., Ohkura, R., and Sutoh, K. (2007). The coordination of cyclic microtubule association/dissociation and tail swing of cytoplasmic dynein. Proc. Natl. Acad. Sci. U.S.A. 104, 16134-16139. doi: 10.1073/pnas.0702370104

Kanaan, N. M., Pigino, G. F., Brady, S. T., Lazarov, O., Binder, L. I., and Morfini, G. A. (2013). Axonal degeneration in Alzheimer's disease: when signaling abnormalities meet the axonal transport system. Exp. Neurol. 246, 44-53. doi: 10.1016/j.expneurol.2012.06.003

Karki, S., and Holzbaur, E. L. (1995). Affinity chromatography demonstrates a direct binding between cytoplasmic dynein and the dynactin complex. J. Biol. Chem. 270, 28806-28811. doi: 10.1074/jbc.270.48.28806

Karki, S., Tokito, M. K., and Holzbaur, E. L. (2000). A dynactin subunit with a highly conserved cysteine-rich motif interacts directly with Arp1. J. Biol. Chem. 275, 4834-4839. doi: 10.1074/jbc.275.7.4834

Kikkawa, M. (2013). Big steps toward understanding dynein. J. Cell Biol. 202, 15-23. doi: 10.1083/jcb.201304099

Kim, J., Hsia, E. Y., Sever, N., Beachy, P. A., and Zheng, X. (2014). Simultaneous measurement of smoothened entry into and exit from the primary cilium. PLoS ONE 9:e104070. doi: 10.1371/journal.pone.0104070

Kimura, A., and Onami, S. (2005). Computer simulations and image processing reveal length-dependent pulling force as the primary mechanism for C. elegans male pronuclear migration. Dev. Cell 8, 765-775. doi: 10.1016/j.devcel.2005.03.007

King, S. J., and Schroer, T. A. (2000). Dynactin increases the processivity of the cytoplasmic dynein motor. Nat. Cell Biol. 2, 20-24. doi: 10.1038/71338

Kon, T., Mogami, T., Ohkura, R., Nishiura, M., and Sutoh, K. (2005). ATP hydrolysis cycle-dependent tail motions in cytoplasmic dynein. Nat. Struct. Mol. Biol. 12, 513-519. doi: 10.1038/nsmb930

Kon, T., Oyama, T., Shimo-Kon, R., Imamula, K., Shima, T., Sutoh, K., et al. (2012). The 2.8 A crystal structure of the dynein motor domain. Nature 484, 345-350. doi: 10.1038/nature10955

Lein, P. J., Banker, G. A., and Higgins, D. (1992). Laminin selectively enhances axonal growth and accelerates the development of polarity by hippocampal neurons in culture. Brain Res. Dev. Brain Res. 69, 191-197. doi: 10.1016/01653806(92)90159-T

Lin, S., Liu, M., Mozgova, O. I., Yu, W., and Baas, P. W. (2012). Mitotic motors coregulate microtubule patterns in axons and dendrites. J. Neurosci. 32, 14033-14049. doi: 10.1523/JNEUROSCI.3070-12.2012

Liu, X., Kapoor, T. M., Chen, J. K., and Huse, M. (2013). Diacylglycerol promotes centrosome polarization in $\mathrm{T}$ cells via reciprocal localization of dynein and myosin II. Proc. Natl. Acad. Sci. U.S.A. 110, 11976-11981. doi: 10.1073/pnas.1306180110

Lu, W., Fox, P., Lakonishok, M., Davidson, M. W., and Gelfand, V. I. (2013). Initial neurite outgrowth in Drosophila neurons is driven by kinesin-powered microtubule sliding. Curr. Biol. 23, 1018-1023. doi: 10.1016/j.cub.2013.04.050

Lye, R. J., Porter, M. E., Scholey, J. M., and McIntosh, J. R. (1987). Identification of a microtubule-based cytoplasmic motor in the nematode C. elegans. Cell 51, 309-318. doi: 10.1016/0092-8674(87)90157-7

Mallik, R., Carter, B. C., Lex, S. A., King, S. J., and Gross, S. P. (2004). Cytoplasmic dynein functions as a gear in response to load. Nature 427, 649-652. doi: 10.1038 /nature02293

Markus, S. M., Punch, J. J., and Lee, W. L. (2009). Motor- and tail-dependent targeting of dynein to microtubule plus ends and the cell cortex. Curr. Biol. 19, 196-205. doi: 10.1016/j.cub.2008.12.047

Mazel, T., Biesemann, A., Krejczy, M., Nowald, J., Muller, O., and Dehmelt, L. (2013). Direct observation of microtubule pushing by cortical dynein in living cells. Mol. Biol. Cell. 25, 95-106. doi: 10.1091/mbc.E13-07-0376

McKenney, R. J., Vershinin, M., Kunwar, A., Vallee, R. B., and Gross, S. P. (2010). LIS1 and NudE induce a persistent dynein force-producing state. Cell 141, 304-314. doi: 10.1016/j.cell.2010.02.035

Melemedjian, O. K., Tillu, D. V., Moy, J. K., Asiedu, M. N., Mandell, E. K., Ghosh, S., et al. (2014). Local translation and retrograde axonal transport of CREB regulates IL-6-induced nociceptive plasticity. Mol. Pain 10:45. doi: 10.1186/1744-8069-10-45

Miller, K. E., DeProto, J., Kaufmann, N., Patel, B. N., Duckworth, A., and van Vactor, D. (2005). Direct observation demonstrates that Liprin- $\alpha$ is required for trafficking of synaptic vesicles. Curr. Biol. 15, 684-689. doi: 10.1016/j.cub.2005.02.061

Myers, K. A., Tint, I., Nadar, C. V., He, Y., Black, M. M., and Baas, P. W. (2006). Antagonistic forces generated by cytoplasmic dynein and myosin-II during growth cone turning and axonal retraction. Traffic 7, 1333-1351. doi: 10.1111/j.1600-0854.2006.00476.x

Nadar, V. C., Ketschek, A., Myers, K. A., Gallo, G., and Baas, P. W. (2008). Kinesin-5 is essential for growth-cone turning. Curr. Biol. 18, 1972-1977. doi: 10.1016/j.cub.2008.11.021

Neuwald, A. F., Aravind, L., Spouge, J. L., and Koonin, E. V. (1999). AAA+: a class of chaperone-like ATPases associated with the assembly, operation, and disassembly of protein complexes. Genome Res. 9, 27-43. doi: 10.1101/gr.9.1.27

Nguyen-Ngoc, T., Afshar, K., and Gonczy, P. (2007). Coupling of cortical dynein and $\mathrm{G}$ alpha proteins mediates spindle positioning in Caenorhabditis elegans. Nat. Cell Biol. 9, 1294-1302. doi: 10.1038/ncb1649

O'connell, C. B., and Wang, Y. L. (2000). Mammalian spindle orientation and position respond to changes in cell shape in a dynein-dependent fashion. Mol. Biol. Cell 11, 1765-1774. doi: 10.1091/mbc.11.5.1765

Paschal, B. M., Shpetner, H. S., and Vallee, R. B. (1987). MAP 1C is a microtubuleactivated ATPase which translocates microtubules in vitro and has dynein-like properties. J. Cell Biol. 105, 1273-1282. doi: 10.1083/jcb.105.3.1273

Paschal, B. M., and Vallee, R. B. (1987). Retrograde transport by the microtubuleassociated protein MAP 1C. Nature 330, 181-183. doi: 10.1038/330181a0

Pfister, K. K. (1999). Cytoplasmic dynein and microtubule transport in the axon: the action connection. Mol. Neurobiol. 20, 81-91. doi: 10.1007/BF02742435

Pfister, K. K. (2015). Distinct functional roles of cytoplasmic dynein defined by the intermediate chain isoforms. Exp. Cell Res. 334, 54-60. doi: 10.1016/j.yexcr.2014.12.013

Pilling, A. D., Horiuchi, D., Lively, C. M., and Saxton, W. M. (2006). Kinesin-1 and Dynein are the primary motors for fast transport of mitochondria in Drosophila motor axons. Mol. Biol. Cell 17, 2057-2068. doi: 10.1091/mbc.E05-06-0526

Porter, M. E., and Johnson, K. A. (1983). Transient state kinetic analysis of the ATP-induced dissociation of the dynein-microtubule complex. J. Biol. Chem. 258, 6582-6587.

Qiu, W., Derr, N. D., Goodman, B. S., Villa, E., Wu, D., Shih, W., et al. (2012). Dynein achieves processive motion using both stochastic and coordinated stepping. Nat. Struct. Mol. Biol. 19, 193-200. doi: 10.1038/nsmb.2205

Reck-Peterson, S. L., Yildiz, A., Carter, A. P., Gennerich, A., Zhang, N., and Vale, R. D. (2006). Single-molecule analysis of dynein processivity and stepping behavior. Cell 126, 335-348. doi: 10.1016/j.cell.2006.05.046

Roberts, A. J., Kon, T., Knight, P. J., Sutoh, K., and Burgess, S. A. (2013). Functions and mechanics of dynein motor proteins. Nat. Rev. Mol. Cell Biol. 14, 713-726. doi: $10.1038 / \mathrm{nrm} 3667$

Roberts, A. J., Malkova, B., Walker, M. L., Sakakibara, H., Numata, N., Kon, T., et al. (2012). ATP-driven remodeling of the linker domain in the dynein motor. Structure 20, 1670-1680. doi: 10.1016/j.str.2012.07.003

Roberts, A. J., Numata, N., Walker, M. L., Kato, Y. S., Malkova, B., Kon, T., et al. (2009). AAA + Ring and linker swing mechanism in the dynein motor. Cell 136, 485-495. doi: 10.1016/j.cell.2008.11.049

Roossien, D. H., Lamoureux, P., and Miller, K. E. (2014). Cytoplasmic dynein pushes the cytoskeletal meshwork forward during axonal elongation. J. Cell Sci. 127, 3593-3602. doi: 10.1242/jcs. 152611

Rusan, N. M., Tulu, U. S., Fagerstrom, C., and Wadsworth, P. (2002). Reorganization of the microtubule array in prophase/prometaphase requires cytoplasmic dynein-dependent microtubule transport. J. Cell Biol. 158, 997-1003. doi: 10.1083/jcb.200204109

Sainath, R., and Gallo, G. (2014). The dynein inhibitor Ciliobrevin D inhibits the bidirectional transport of organelles along sensory axons and impairs NGFmediated regulation of growth cones and axon branches. Dev. Neurobiol. 75, 757-777. doi: 10.1002/dneu.22246

Sale, W. S., and Satir, P. (1977). Direction of active sliding of microtubules in Tetrahymena cilia. Proc. Natl. Acad. Sci. U.S.A. 74, 2045-2049.

Schlager, M. A., Serra-Marques, A., Grigoriev, I., Gumy, G. F., Esteves da Silva, M., Wulf, P. S., et al. (2014). Bicaudal d family adaptor proteins 
control the velocity of Dynein-based movements. Cell Rep. 8, 1248-1256. doi: 10.1016/j.celrep.2014.07.052

Schmidt, D. J., Rose, D. J., Saxton, W. M., and Strome, S. (2005). Functional analysis of cytoplasmic dynein heavy chain in Caenorhabditis elegans with fast-acting temperature-sensitive mutations. Mol. Biol. Cell 16, 1200-1212. doi: 10.1091/mbc.E04-06-0523

Schnapp, B. J., and Reese, T. S. (1989). Dynein is the motor for retrograde axonal transport of organelles. Proc. Natl. Acad. Sci. U.S.A. 86, 1548-1552.

Schroer, T. A. (2004). Dynactin. Annu. Rev. Cell Dev. Biol. 20, 759-779. doi: 10.1146/annurev.cellbio.20.012103.094623

Schroer, T. A., and Sheetz, M. P. (1991). Two activators of microtubule-based vesicle transport. J. Cell Biol. 115, 1309-1318. doi: 10.1083/jcb.115.5.1309

Schroer, T. A., Steuer, E. R., and Sheetz, M. P. (1989). Cytoplasmic dynein is a minus end-directed motor for membranous organelles. Cell 56, 937-946. doi: 10.1016/0092-8674(89)90627-2

Shih, S. M., Engel, B. D., Kocabas, F., Bilyard, T., Gennerich, A., Marshall, W. F., et al. (2013). Intraflagellar transport drives flagellar surface motility. Elife 2:e00744. doi: 10.7554/eLife.00744

Sikirzhytski, V., Magidson, V., Steinman, J. B., He, J., Le Berre, M., Tikhonenko, I., et al. (2014). Direct kinetochore-spindle pole connections are not required for chromosome segregation. J. Cell Biol. 206, 231-243. doi: 10.1083/jcb.201401090

Spillane, M., Ketschek, A., Donnelly, C. J., Pacheco, A., Twiss, J. L., and Gallo, G. (2012). Nerve growth factor-induced formation of axonal filopodia and collateral branches involves the intra-axonal synthesis of regulators of the actin-nucleating Arp2/3 complex. J. Neurosci. 32, 17671-17689. doi: 10.1523/JNEUROSCI.1079-12.2012

Torisawa, T., Nakayama, A., Furuta, K., Yamada, M., Hirotsune, S., and Toyoshima, Y. Y. (2011). Functional dissection of LIS1 and NDEL1 towards understanding the molecular mechanisms of cytoplasmic dynein regulation. J. Biol. Chem. 286, 1959-1965. doi: 10.1074/jbc.M1110.169847

Toropova, K., Zou, S., Roberts, A. J., Redwine, W. B., Goodman, B. S., ReckPeterson, S. L., et al. (2014). Lis1 regulates dynein by sterically blocking its mechanochemical cycle. Elife 3:e03372. doi: 10.7554/eLife.03372

Vale, R. D. (2003). The molecular motor toolbox for intracellular transport. Cell 112, 467-480. doi: 10.1016/S0092-8674(03)00111-9

Valetti, C., Wetzel, D. M., Schrader, M., Hasbani, M. J., Gill, S. R., Kreis, T. E., et al. (1999). Role of dynactin in endocytic traffic: effects of dynamitin overexpression and colocalization with CLIP-170. Mol. Biol. Cell 10, 4107-4120. doi: $10.1091 / \mathrm{mbc}$.10.12.4107

Vallee, R. B., McKenney, R. J., and Ori-McKenney, K. M. (2012). Multiple modes of cytoplasmic dynein regulation. Nat. Cell Biol. 14, 224-230. doi: $10.1038 /$ ncb2420

Vaughan, K. T., Tynan, S. H., Faulkner, N. E., Echeverri, C. J., and Vallee, R. B. (1999). Colocalization of cytoplasmic dynein with dynactin and CLIP-170 at microtubule distal ends. J. Cell Sci. 112(Pt 10), 1437-1447.
Vaughan, K. T., and Vallee, R. B. (1995). Cytoplasmic dynein binds dynactin through a direct interaction between the intermediate chains and p150Glued. J. Cell Biol. 131, 1507-1516. doi: 10.1083/jcb.131.6.1507

Vaughan, P. S., Miura, P., Henderson, M., Byrne, B., and Vaughan, K. T. (2002). A role for regulated binding of p150(Glued) to microtubule plus ends in organelle transport. J. Cell Biol. 158, 305-319. doi: 10.1083/jcb.200201029

Wang, H. M., Yan, Q., Yang, T., Cheng, H., Du, J., Yoshioka, K., et al. (2015). Scaffold protein JLP is critical for CD40 signaling in B lymphocytes. J. Biol. Chem. 290, 5256-5266. doi: 10.1074/jbc.M114.618496

Waterman-Storer, C. M., Karki, S., and Holzbaur, E. L. (1995). The p150Glued component of the dynactin complex binds to both microtubules and the actinrelated protein centractin (Arp-1). Proc. Natl. Acad. Sci. U.S.A. 92, 1634-1638.

Wittmann, T., and Hyman, T. (1999). Recombinant p50/dynamitin as a tool to examine the role of dynactin in intracellular processes. Methods Cell Biol. 61, 137-143.

Wuhr, M., Tan, E. S., Parker, S. K., Detrich, H. W. III, and Mitchison, T. J. (2010). A model for cleavage plane determination in early amphibian and fish embryos. Curr. Biol. 20, 2040-2045. doi: 10.1016/j.cub.2010.10.024

Yamada, M., Toba, S., Yoshida, Y., Haratani, K., Mori, D., Yano, Y., et al. (2008). LIS1 and NDEL1 coordinate the plus-end-directed transport of cytoplasmic dynein. EMBO J. 19, 2471-2483. doi: 10.1038/emboj.20 08.182

Ye, F., Breslow, D. K., Koslover, E. F., Spakowitz, A. J., Nelson, W. J., and Nachury, M. V. (2013). Single molecule imaging reveals a major role for diffusion in the exploration of ciliary space by signaling receptors. Elife 2:e00654. doi: 10.7554/eLife.00654

Yi, J., Wu, X., Chung, A. H., Chen, J. K., Kapoor, T. M., and Hammer, J. A. (2013). Centrosome repositioning in $\mathrm{T}$ cells is biphasic and driven by microtubule end-on capture-shrinkage. J. Cell Biol. 202, 779-792. doi: 10.1083/jcb.2013 01004

Yi, J. Y., Ori-McKenney, K. M., Mckenney, R. J., Vershinin, M., Gross, S. P., and Vallee, R. B. (2011). High-resolution imaging reveals indirect coordination of opposite motors and a role for LIS1 in high-load axonal transport. J. Cell Biol. 195, 193-201. doi: 10.1083/jcb.201104076

Conflict of Interest Statement: The authors declare that the research was conducted in the absence of any commercial or financial relationships that could be construed as a potential conflict of interest.

Copyright (C) 2015 Roossien, Miller and Gallo. This is an open-access article distributed under the terms of the Creative Commons Attribution License (CC BY). The use, distribution or reproduction in other forums is permitted, provided the original author(s) or licensor are credited and that the original publication in this journal is cited, in accordance with accepted academic practice. No use, distribution or reproduction is permitted which does not comply with these terms. 